\title{
Touch and Feel Soft Hardware
}

Ylva Fernaeus

Mobile Life Centre and

Royal Institute of Technology

Sweden

fernaeus@kth.se

\section{Anna Vallgårda}

The Swedish School of Textiles,

University of Borås

Sweden

anna.vallgarda@hb.se

\section{Mili John Tharakan}

The Swedish School of Textiles,

University of Borås

Sweden

mili.tharakan@hb.se

\section{Anders Lundström}

Royal Institute of Technology

Sweden

andelund@kth.se

\begin{abstract}
With soft hardware we refer to electronic components, coatings, and shells built from materials that make them elastic, flexible, floppy and malleable. By introducing new material properties into electronic and computational contexts we expect to open new paths for designing interactive things. Building electronics with textile and other soft materials may easily degrade elements such as speed, power, and storage capacities; however, these constraints can be acceptable if not down right desirable in these new contexts. We see how sensors, actuators, computers and even battery cells made of soft materials enables us to embed them into soft shapes that in turn afford certain forms of interaction. With the term soft hardware, we also highlight the interplay between computational and physical materials in interaction designs.
\end{abstract}

\section{B Detailed proposal description}

In this studio we will invite participants to bring and work hands-on with soft hardware in an exploratory open-ended session, with a special focus on tactile experiences.

Beside various conductive fabrics, yarns, and other soft conductive components we will bring some example projects. For example, a carpet Patcher (See Figure 1), simple e-embroidery samples (See Figure 2), a stretch and squeeze interaction device (See Figure 3), a 


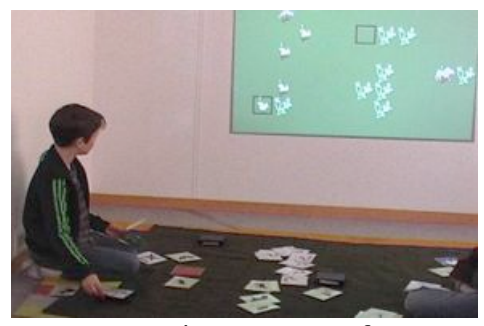

Figure 1 Patcher. a carpet for making animated games.

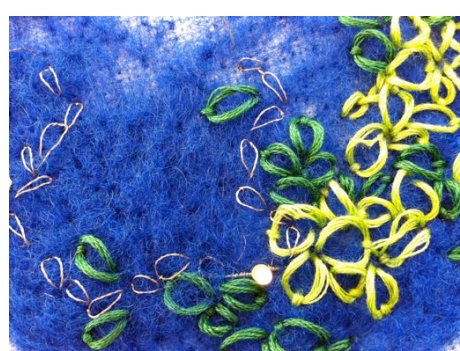

Figure 2 Example of an eembroidery

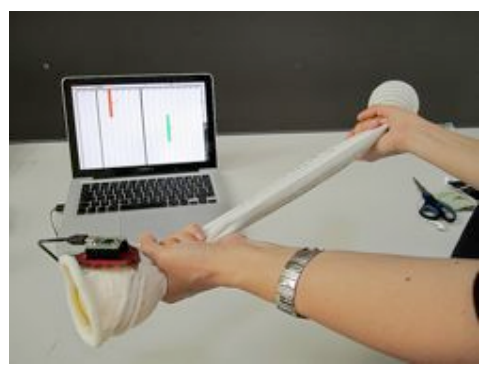

Figure $3 \mathrm{~A}$ rough prototype of a stretch and squeeze interaction device. thermo chromic ink test platform for textile printing (See Figure 4), ActDresses to control a robots behavior (See Figure 5) Rolling stones, a series of robotic balls covered in different traditional textile techniques (See Figure 6). Also, participants are encouraged to bring materials, projects, or technologies that they find relevant or interesting for this topic.

The discussions will concern the experiential qualities of soft materials in interaction design, and particular challenges, lessons learned, and suggested approaches that the participants like to share.

Designs based on soft hardware have over the recent decades repeatedly been showcased with much interest from the general public, for example, the digital paper [11], interactive displays on curtains and wallpapers [4, 5], interactive carpets, furniture hooked up to the internet [9], robotic plush toys and clothes with embedded electronics $[2,10]$. Many quite remarkable prototypes have been produced, but the full potential of technology based on soft hardware has not yet been realized either in products or large-scale productions. Indeed, many of us, even in the domain of TEI, still lack personal hands on experiences of how most of these artifacts actually feel like. Still, we would like to emphasize that soft hardware is now one of the main themes of TEI, especially in terms of crafting with new materials. Examples include Leah Buechley et al's work with the textile LilyPad toolkit [3] and kit of no parts [8], Seed studios soft Seeduino film arduino board (handed out to participants at TEI'11), and a constantly growing set of prototypes and concepts showcased as demos. At last year's TEI conference, where the student design competition took the form of a fashion show, an impressive range of wearable interactive costumes were showcased. We therefore believe that TEI is a perfect arena for fruitful discussions around the theme of soft hardware.

\section{Program for the studio}

After a quick lunch and brief introductions, we will initiate a simple hands-on exercise with soft hardware that participants can work on during subsequent presentations and discussions.

In a "show and tell" session participants will then presents their own soft objects, materials, or something curious they happened to come upon within the area of soft hardware. Everyone should bring something, either something they fabricated themselves, or something ready made.

We will end the day with a discussions of two-three central questions either raised through the "show-andtell" session or from this proposal (See 1.C Topics to be Covered). The point is to find out where we can go from here in terms of a continuous knowledge exchange and possibly collaborations.

\section{C Topics to be covered}

The purpose of this studio is to make use of the physical gathering of people in this domain. Together we will develop our understanding both through each other's experiences with new materials in electronic contexts and through our common hands-on explorations of soft hardware at the workshop. Participants without personal experience of working with soft hardware are also welcome. Every participant is encouraged to bring a soft hardware artifact they are working on or one that hold interesting tactile properties or in other ways could fit into the theme. 


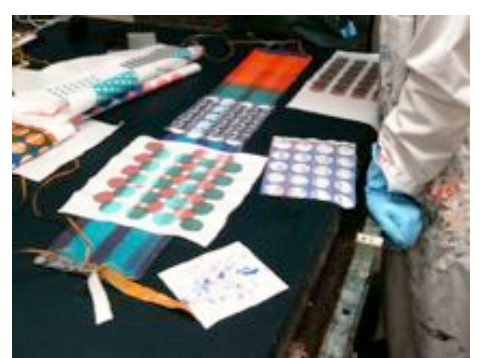

The focus of this studio will be on the practical challenges that designers often experience in their efforts to realize products with soft hardware, for example:

- Finding balanced solutions where e.g. textile qualities (such as softness, flexibility, elasticity, washable) are not lost at the expense of the limitations of electronic components.

Figure 4 Test platform for thermo chromic textile printing

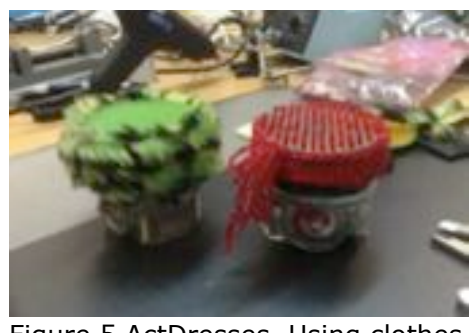

Figure 5 ActDresses. Using clothes to control software of robots.

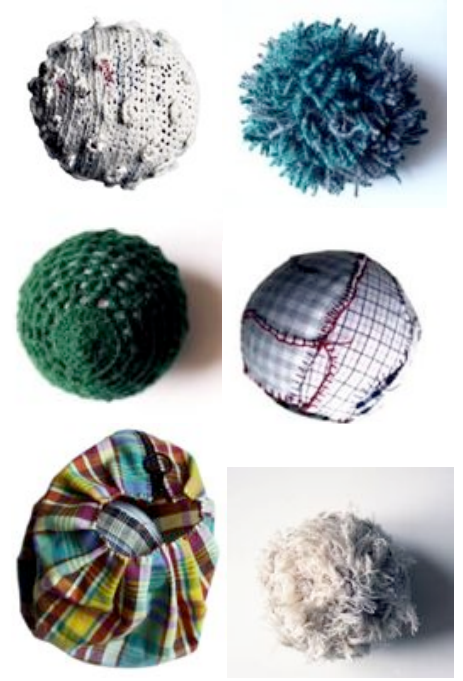

Figure 6 Rolling stones, a series of robotic balls covered in different traditional textile techniques.

\section{D Learning goals/Discussion objectives}

The idea of soft hardware may be used to highlight the interplay between computations and materiality, tying to concepts such as atoms and bits [7], and as more recently revisited by several others (e.g. $[1,6,12]$ ).
Soft hardware, however, need not remain in the conceptual realm but can become this repertoire of soft electronic or computational components, which can engage new forms of interaction exactly because of its material affordances. These components may not be as powerful or precise as their hard counterparts but that may be ok because they have other qualities-textile qualities.

Textiles lend us a myriad of techniques, tools, material properties, scales and structures making it a natural choice for creating soft hardware. The accommodating and adaptable properties of textiles are its greatest strength and the reason for its use in fields outside of itself such as medical textiles, transport and geotextiles. Textile craft has a history as old as mankind itself and the depth of knowledge about 'making', which is found within this craft, is built on generations of makers. We can adapt this knowledge and skills to produce better design solutions, create new expressions and aesthetics in interaction design projects. Soft hardware still remains very much within the handcraft domain and therefore the 'making' skills need to be developed and refined into that of a craft.

Designing things from soft hardware, however, incorporate several knowledge domains that are traditionally quite disparate, including electronics, textile craftsmanship, and practices of interaction design and programming. Practices around how to work in this new design discipline are not yet established, and our experience is that there is a great demand for interdisciplinary knowledge exchange. The main purpose of the studio is therefore to open for such cross-disciplinary discussions based on the various 
perspectives that participants bring from their respective backgrounds.

\section{E References}

[1] Blanchette, J.-F. 2011. A Material History of Bits. Journal of the American Society for Information Science and Technology. 62, 6, 1042-1057.

[2] Braddock-Clarke, S. E. and O'Mahony, M. 2006 TECHNO TEXTILES 2 Revolutionary Fabrics for Fashion and Design. New York, NY: Thames and Hudson.

[3] Buechley, L., Eisenberg, M., Catchen, J., and Crockett, A. 2008. The LilyPad Arduino: using computational textiles to investigate engagement aesthetics, and diversity in computer science education. In proceedings of the twenty-sixth annual SIGCHI conference on Human factors in computing systems (CHI '08), Florence, Italy.

[4] Coelho, M. 2009. Shutters: A Permeable Surface for Environmental Control and Communication. In proceedings of the Third International Conference on Tangible and Embedded Interaction (TEI'09), Feb 1618, Cambridge, UK.

[5] Coelho, M., Hall, L., Berzowska, J., and Maes, P. 2009. Pulp-Based Computing: A Framework for Building Computers Out of Paper. In proceedings of the Conference on Human Factors in Computing Systems, April 4-9, Boston, MA, USA.

[6] Dourish, P. and Mazmanian, M. 2011. Media as Material: Information Representations as Material

Foundations for Organizational Practice. In proceedings of the Working Paper for the Third International Symposium on Process Organization Studies, Corfu, Greece.

[7] Ishii, H. and Ullmer, B. 1997. Tangible Bits: Towards Seamless Interfaces between People, Bits and Atoms. In proceedings of the Conference on Human Factors in Computing Systems (CHI'97), Atlanta, GA.
[8] Perner-Wilson, H., Buechley, L., and Satomi, M. 2010. Handcrafting textile interfaces from a kit-of-noparts. In proceedings of the fifth international conference on Tangible, embedded, and embodied interaction (TEI '11), Funchal, Portugal.

[9] Redström, M., Redström, J., and Mazé, R. (Eds.) 2005. IT+Textiles. Helsinki, Finland: Edita/IT Press.

[10] Seymore, S. (Ed.) 2008. Fashionable Technology. Vienna, Austria: Springer.

[11] Signer, B. and Norrie, M. C. 2010. Interactive Paper: Past, Present and Future. In proceedings of the 1st International Workshop on Paper Computing (PaperComp 2010), Copenhagen, Denmark.

[12] Vallgårda, A. and Redström, J. 2007.

Computational Composites. In proceedings of the Conference on Human Factors in Computing Systems, April 28 - May 3, San José, USA.

\section{Acknowledgements}

We would like to thank our collaborators in the Wireless Textile project, especially Linda Worbin and Kristian Karlsson. We would also like to thank Vinnova Vinvext, for the funding the Smart Textile research program through which many of our included projects have been carried out. 